\title{
A chicken-or-egg conundrum in apoptosis: which comes first? Ceramide or PKC $\delta$ ?
}

Commentary

See related article, pages $827-836$.

\author{
Steven Grant ${ }^{1,2}$ and Sarah Spiegel ${ }^{2}$ \\ ${ }^{1}$ Department of Medicine, and \\ ${ }^{2}$ Department of Biochemistry, Medical College of Virginia Campus, Virginia Commonwealth University, Richmond, Virginia, USA \\ Address correspondence to: Sarah Spiegel, Department of Biochemistry, Virginia Commonwealth University, \\ 1101 East Marshall Street, Sanger Hall Room 2-011, PO Box 980614, Richmond, Virginia 23298-0614, USA. \\ Phone: (804) 828-9330; Fax: (804) 828-8999; E-mail: sspiegel@mail1.vcu.edu.
}

J. Clin. Invest. 109:717-719 (2002). DOI:10.1172/JCI200215302.

Recent intense interest has focused on the activation of the mitochondrial pathway to apoptosis, particularly as it occurs in tumor cells treated with cytotoxic agents such as doxorubicin and etoposide. The contribution of two prominent molecular players in this response, the sphingolipid second messenger ceramide and the novel protein kinase $\mathrm{C}$ isoform (PKC $\delta$ ), are only now being appreciated, but their significance and their interactions with one another remain controversial. While some studies argue strongly that ceramide generation is critical to apoptosis induced by certain drugs $(1,2)$, others have cast doubt on this notion $(3,4)$.

A variety of stress stimuli, as well as radiation and anti-cancer drugs, generate ceramide through the sphingomyelinase (SMase) pathway (Figure
1). SMases are analogous in their action to phospholipases $\mathrm{C}$, but act specifically on the ubiquitous membrane phospholipid sphingomyelin. Neutral and acidic forms of SMase (distinguishable by their $\mathrm{pH}$ optima) have been described, and both are implicated in the induction of apoptosis $(5,6)$. Another, de novo route to ceramide biosynthesis may also be involved in this process $(1,2,7)$. Here, serine pamitoyl-transferase catalyzes the condensation of serine and palmitoyl-CoA to form 3-ketosphinganine, which is reduced to sphinganine and subsequently $N$-acylated by ceramide synthase to form dihydroceramide. Finally, a double bond is introduced by dihydroceramide desaturase to produce ceramide ( $N$-acyl sphingosine). However it is formed, accumulating ceramide apparently activates cytochrome $c$ release, caspase activation, and apoptosis $(5,6)$; the direct targets of ceramide in this pathway are still a matter of debate.

Many groups have also demonstrated that induction of apoptosis by cytotoxic drugs or by phorbol esters is associated with translocation of PKC $\delta$ to the mitochondria, accompanied by cytochrome $c$ release and activation of the apoptotic cascade (8-11). In some systems, interference with PKC $\delta$ translocation/activation has been shown to attenuate druginduced lethality (8-10). Again, however, the functional significance of these events in the apoptotic process remains to be fully defined (12).

The relationship between ceramide and PKC $\delta$ is highly complex, with evidence that ceramide generation can represent either a cause $(13,14)$ or a consequence (7) of PKC $\delta$ activation. Efforts to reconcile these disparate

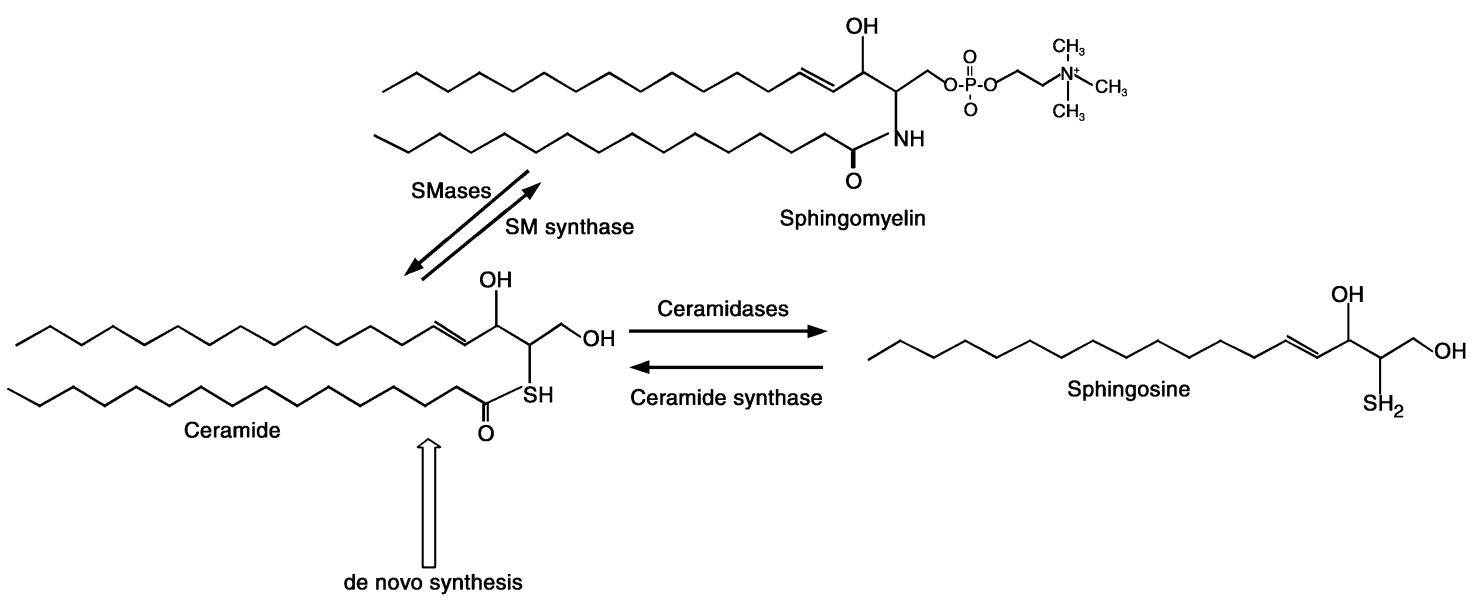

\section{Figure 1}

Formation of ceramide in mammalian cells. Many stress stimuli, including anticancer drugs, $\gamma$-radiation, ultraviolet, cytokines, and growth factor withdrawal, activate sphingomyelinases, which remove the head group from the membrane phospholipid sphingomyelin, forming ceramide. An opposing reaction, catalyzed by sphingomyelin synthase, occurs when phosphocholine is transferred from phosphatidylcholine to the primary hydroxyl of ceramide, producing sphingomyelin and diacylglycerol. In addition, ceramide can be produced through a de novo biosynthetic pathway, described in the text. Sphingosine is not produced by the de novo pathway and can only be formed by deacylation of ceramide catalyzed by ceramidases. 
findings have been hampered by the lack of a unified model system in which to define the interactions between ceramide and $\mathrm{PKC} \delta$ and explore their roles in regulating cell death. In a report appearing in this issue of the JCI, Sumitomo and colleagues provide a conceptual model that might help to clarify these issues (15). These authors show that in LNCaP prostate cancer cells, which express PKC $\delta$, exposure to cytotoxic drugs such as etoposide and paclitaxel induces a biphasic increase in ceramide synthesis. In the initial phase, transient increases in ceramide levels stem from stimulation of the de novo synthetic pathway. Subsequent, sustained increases in ceramide levels, however, depend on neutral sphingomyelinase activation and sphingomyelin hydrolysis. Significantly, the latter process appears to be dependent upon caspase- 9 activation, which in turn represents a consequence of translocation of PKC $\delta$ to the mitochondria and release of the proapoptogenic protein cytochrome c. In this way, the generation of ceramide and the translocation of PKC $\delta$ to the mitochondria - resulting from drug treatment - cooperate to amplify apoptotic signals, thereby ensuring complete activation of the cell death cascade.

Aside from providing insights into the mechanism by which cytotoxic drugs induce prostate cancer cell death, these observations could also help to reconcile conflicting findings in literature regarding the hierarchal roles of ceramide and PKC $\delta$ in apoptotic events. For example, the finding of an initial, transient increase in de novo ceramide synthesis in drugtreated cells is entirely compatible with the notion that upstream-generated ceramide is responsible for PKC $\delta$ translocation $(13,14)$. On the other hand, the evidence that druginduced PKC $\delta$ translocation and activation plays a functional role in the caspase-9-mediated generation of ceramide and potentiation of apoptosis is consistent with reports indicating that activation of PKC $\delta$ by phorbol esters in LNCaP prostate cancer cells contributes to ceramide formation (7). The complexity of this feedback amplification loop is underscored by the observation that caspase-9 inhibition also attenuates
PKC $\delta$ mitochondrial translocation, implying that $\mathrm{PKC} \delta$ can promote its own activation through a ceramidemediated pro-cess. Similarly, induction of apoptosis by cross-linking of Fas triggers the biphasic generation of ceramide, which is then converted to sphingosine. Along with ceramide and released cytochrome $c$, sphingosine itself can activate caspase- 8 and the proapoptotic $\mathrm{Bcl}-2$ family member, Bid. Other proapoptotic molecules, perhaps the mitochondrionderived, apoptosis-inducing factor (AIF), may be required to amplify this process (16). This would not be the first example of an amplification loop sealing the fate of tumor cells following cytotoxic drug exposure. Drug-induced cytochrome $c$ release can result in caspase-3 activation, leading in turn to the secondary cleavage of prolapses- 8 , Bid activation and mitochondrial translocation, and further mitochondrial injury (17).

A number of questions remain to be answered before the role of ceramide and PKC $\delta$ in apoptosis can be more fully understood. For instance, recent studies suggest that $\mathrm{Bcl}-2$ and $\mathrm{Bcl}-\mathrm{xL}$ act by disrupting interactions between Bcl-2 homology domain-3 (BH3), domain-only family members and multidomain pro-apoptotic proteins such as Bax and Bak to trigger cytochrome $c$ release (18). Questions therefore arise as to how, whether, and at what level ceramide and PKC $\delta$ might interact with these proteins to promote mitochondrial injury and initiation of the apoptotic caspase cascade. How does ceramide, synthesized in the endoplasmic reticulum, regulate translocation of $\mathrm{PKC} \delta$ to the mitochondria? There is some evidence suggesting that ceramide might bind directly to PKC $\delta$ (19), but it is also possible that the ability of ceramide to form platforms of sphingolipidenriched membrane domains (20) is involved in translocation of PKC $\delta$ to the mitochondria.

Another important question concerns the identity of the neutral SMases activated by caspase- 9 , as the relevant molecule has not yet been cloned. In addition, even if ceramide and PKC $\delta$ can be shown to play key roles in drug-induced apoptosis in certain cells, other forms of nonapoptotic cell death have been described. For this reason, cytotoxic drugs might inhibit self-renewal even in tumor cells defective in ceramideand/or PKC $\delta$-dependent apoptosis. A final consideration relates to the effects of ceramide generation and $\mathrm{PKC} \delta$ activation on other pro-apoptotic mitochondrial proteins, including AIF (21) and Smac/DIABLO, which antagonize the actions of inhibitor of apoptosis proteins (22). In this context, it is interesting that a recent study (23) suggests that release of Smac/DIABLO is required for activation of the caspase cascade by cytochrome $c$ in prostate cancer cells. Answers to these questions are greatly needed to deepen our understanding of apoptosis and to inspire new approaches for treating prostate cancer or other malignancies.

1. Bose, R., et al. 1995. Ceramide synthase mediates daunorubicin-induced apoptosis: an alternative mechanism for generating death signals. Cell. 82:405-414.

2. Perry, D.K., et al. 2000. Serine palmitoyltransferase regulates de novo ceramide generation during etoposide-induced apoptosis. J. Biol. Chem. 275:9078-9084.

3. Hofmann, K., and Dixit, V.M. 1998. Ceramide in apoptosis-does it really matter? Trends Biochem. Sci. 23:374-377.

4. Tepper, A.D., de Vries, E., van Blitterswijk, W.J. and Borst, J. 1999. Ordering of ceramide formation, caspase activation, and mitochondrial changes during CD95- and DNA damageinduced apoptosis. J. Clin. Invest. 103:971-978.

5. Kolesnick, R., and Hannun, Y.A. 1999. Ceramide and apoptosis. Trends Biochem. Sci. 24:224-225.

6. Hannun, Y.A., and Luberto, C. 2000. Ceramide in the eukaryotic stress response. Trends Cell Biol. 10:73-80.

7. Garzotto, M., et al. 1998. 12-O-tetradecanoylphorbol-13-acetate-induced apoptosis in LNCaP cells is mediated through ceramide synthase. Cancer Res. 58:2260-2264.

8. Basu, A., Woolard, M.D., and Johnson, C.L. 2001. Involvement of protein kinase C-delta in DNA damage-induced apoptosis. Cell Death Differ. 8:899-908.

9. Matassa, A.A., Carpenter, L., Biden, T.J., Humphries, M.J., and Reyland, M.E. 2001. PKCdelta is required for mitochondrial-dependent apoptosis in salivary epithelial cells. J. Biol. Chem. 276:29719-29728.

10. Majumder, P.K., et al. 2000. Mitochondrial translocation of protein kinase $\mathrm{C}$ delta in phorbol ester-induced cytochrome c release and apoptosis. J. Biol. Chem. 275:21793-21796.

11. Li, L., Lorenzo, P.S., Bogi, K., Blumberg, P.M., and Yuspa, S.H. 1999. Protein kinase Cdelta targets mitochondria, alters mitochondrial membrane potential, and induces apoptosis in normal and neoplastic keratinocytes when overexpressed by an adenoviral vector. Mol. Cell Biol. 19:8547-8558.

12. Dal Pra, I., Whitfield, J.F., Chiarini, A., and Armato, U. 2000. Increased activity of the protein kinase C-delta holoenzyme in the cytoplasmic particulate fraction precedes the activation of caspases in polyomavirus-transformed pyF111 rat fibroblasts exposed to calphostin C or topoisomerase-II inhibitors. Exp. Cell Res. 255:171-183. 
13. Kajimoto, T., Ohmori, S., Shirai, Y., Sakai, N., and Saito, N. 2001. Subtype-specific translocation of the delta subtype of protein kinase $\mathrm{C}$ and its activation by tyrosine phosphorylation induced by ceramide in HeLa cells. Mol. Cell Biol. 21:1769-1783.

14. Sawai, H., et al. 1997. Ceramide-induced translocation of protein kinase C-delta and -epsilon to the cytosol. Implications in apoptosis. J. Biol. Chem. 272:2452-2458.

15. Sumitomo, M., et al. 2002. Protein kinase C $\delta$ amplifies ceramide formation via mitochondrial signaling in prostate cancer cells. J. Clin. Invest. 109:827-836. DOI:10.1172/JCI200214146.
16. Cuvillier, O., Edsall, L., and Spiegel, S. 2000 Involvement of sphingosine in mitochondriadependent fas-induced apoptosis of type II jurkat T cells. J. Biol. Chem. 275:15691-15700.

17. Sun, X.M., et al. 1999. Distinct caspase cascades are initiated in receptor-mediated and chemical-induced apoptosis. J. Biol. Chem. 274:5053-5060.

18. Cheng, E.H., et al. 2001. BCL-2, BCL-X(L) sequester $\mathrm{BH} 3$ domain-only molecules preventing BAX- and BAK-mediated mitochondrial apoptosis. Mol. Cell. 8:705-711.

19. Huwiler, A., Fabbro, D., and Pfeilschifter, J. 1998
Selective ceramide binding to protein kinase Calpha and -delta isoenzymes in renal mesangial cells. Biochemistry. 37:14556-14562.

20. Cremesti, A., et al. 2001. Ceramide enables fas to cap and kill. J. Biol. Chem. 276:23954-23961.

21. Susin, S.A., et al. 1999. Molecular characterization of mitochondrial apoptosis-inducing factor Nature. 397:441-446.

22. Wu, G., et al. 2000. Structural basis of IAP recognition by Smac/DIABLO. Nature. 408:1008-1012.

23. Carson, J.P., et al. 2002. Smac is required for cytochrome c-induced apoptosis in prostate cancer LNCaP cells. Cancer Res. 62:18-23. 